\title{
Métricas para Avaliação de Sistemas de Informação
}

\author{
Raquel Dias \\ Instituto de Cooperação e Assistência Técnica da AEUDF - Av. W4 Sul - Q 704 - Brasília - DF \\ raquel dias@directnet.com.br
}

\begin{abstract}
Resumo - O objetivo deste artigo é apresentar um modelo simplificado para avaliação da qualidade de sistema de informação. O modelo está baseado na observação do comportamento do consumidor de bens e serviços, e enfoca os aspectos ambientais que influenciam o processo de tomada de decisão de tais agentes. Este artigo, além de apresentar um modelo capaz de prever demandas futuras de usuários reincidentes, mostra a importância da existência de métricas, objetivas, para o gerenciamento da qualidade durante o processo de desenvolvimento de sistemas de informação, descreve um modelo para avaliação de tais sistemas baseado nos princípios da utilidade, usabilidade e qualidade.
\end{abstract}

\section{Palavras-chave: Métricas , Avaliação de Sistemas, Qualidade de Sistemas de Informação}

\begin{abstract}
The objective of this paper is to propose a simplified model for evaluation of quality of systems of information. The model is based on the observation of the consumer's of goods behavior and services, and it focuses the environmental aspects that influence the process of taking of such agents' decision. This work, besides presenting a model capable to foresee recurrent users' future demands, shows the importance of the existence of metric, objective, for the management of the quality during the process of development of systems of information, and it describes a model for evaluation of such systems based on the beginnings of the usefulness, usability and quality.
\end{abstract}

Key-words: Metrics, Information systems evaluation,quality of information systems

\section{Introdução}

Existem múltiplas dimensões relacionadas com o uso de sistemas de informação que podem ser quantificadas. Pode-se utilizar monitores em rede local, rede servidora ou mainframe e sistema de gerenciamento de rede ou analisador de tráfego quando se define a utilização como uma percentagem de capacidade, ou em termos absolutos, baseada estritamente no número de usuários conectados ao sistema. Pode-se também adotar um modelo de desempenho do sistema usando ferramentas de modelagem. Existem várias ferramentas de simulação para modelos de diferentes níveis de utilização do sistema de informação, e para fazer estimativas do desempenho do sistema em vários níveis de uso ou saturação.

Vários indicadores tradicionais de desempenho de centro de dados podem retratar a qualidade de um sistema de informação, dentre eles destacamos:

- percentual de uso do processador central.

- Quantidade de acessos diretos na unidade de armazenamento .
- Espaço de disco acessado durante determinado período de tempo.

- Uso de fita.

- Uso de impressão.

Outra consideração é simplesmente o desempenho do nível de serviço. Muitos sistemas de informações têm um nível de serviço especificado pela sua disponibilidade para 0 usuário final. A contabilização do tempo em que o sistema ficou indisponível durante aquele período em que deveria estar sendo utilizado pelo usuário final, demonstra uma redução na disponibilidade do sistema e, presumidamente, no seu nível de utilização. É de se esperar, então, que muitas organizações meçam a disponibilidade de seus sistemas de informação estendendo o significado dessas medidas para o nível de utilização. Esta medida no entanto pode mascarar a situação real de saturação do sistema, em termos de concentração de usuários acessando o sistema. Se o sistema está disponível, somente durante um curto período de tempo, a utilização pode ser artificialmente alta, bem superior ao que seria se 0 intervalo de tempo fosse o normal. 
Em resumo, não existe nenhuma medida global que retrate claramente a utilização dos sistemas de informação. A questão tem que ser pré-definida focalizando determinado interesse de pesquisa sobre o sistema, por exemplo hardware, software, redes, e outros, ou o seu foco deverá recair sobre os usuários e usuários em potencial dos sistemas e se eles usam o sistema, como o usam, e porque e quando o usam .

Este artigo ressalta a importância de métricas, objetivas, para o gerenciamento de sistemas de informação, focalizando os usuários finais do sistema. A qualidade das informações bem como a forma em que foram organizadas em um sistema de informações pode ser vista através do grau de aceitação do sistema pelos seus usuários diretos e indiretos. Os usuários diretos, são aqui representados por aqueles diretamente afetados pelo sistema, incluindo os usuários finais, para os quais foram criados os sistemas. Os usuários indiretos, consistem no público em geral e naqueles que demonstram interesse em aperfeiçoar o sistema, incluindo-se nesta categoria os usuários finais voluntários, programadores, analistas, administradores de bases de dados.

\section{Metodologia}

Trata-se de uma pesquisa, exploratória. $\mathrm{O}$ objetivo principal foi o aprimoramento de idéias e a descoberta de intuições. Envolveu, portanto, levantamento bibliográfico, análise de dados relativos aos usuários que tiveram experiências práticas com o problema pesquisado e análise de resultados da aplicação do modelo.

O levantamento bibliográfico teve como objetivo possibilitar a construção de hipóteses, assumindo um caráter de estudo exploratório, além de delinear o estado da arte relacionado ao problema pesquisado. $\mathrm{O}$ resultado culminou com a elaboração de modelo para avaliação de sistemas de informação focalizando o usuário final, conforme discutiremos e descreveremos nas seções seguintes.

\section{Fundamentação Teórica}

Davis, Bagozzi \& Warshow 1989 realizaram um estudo sobre aceitação das tecnologias de tratamento automático da informação onde foram observadas as opiniões dos usuários finais quanto à percepção da utilidade e da facilidade de uso do sistema, identificados como principais indicadores da utilização e satisfação de um sistema de tratamento automático da informação. Estes dois indicadores estariam relacionados diretamente às variáveis ligadas à informações que explicitam claramente os perfis dos usuários. Neste caso tais variáveis controladas pelo sistema ou independentes explicam o comportamento do usuário e suas diferenças individuais. [1] .

Vários aspectos relacionados às hipóteses de avaliação devem ser observados, tais como a facilidade de utilização da ferramenta, que está relacionada com os aspectos funcionais $e$ ergonômicos, a utilidade para o decisor referindose ao impacto da informação ligada à necessidade para resolução dos problemas, o efeito provocado pela freqüente utilização do sistema,o valor do sistema percebido pelo usuário e por fim, a verificação do efeito da não-interrupção da consulta [2]

Ahituy, em 1990, ressaltou a importância da existência da pertinência da informação como um aspecto a medir. A informação processada e que circula deve ser avaliada em termos de utilidade, qualidade e quantidade. De acordo com Ahituy, "o importante é poder avaliar e comparar alternativas quando se está a ponto de tomar uma decisão referente à criação ou à seleção de um sistema de informações", ressaltando que os atributos relacionados com o sistema, tais como tempos, desempenho, dados, bem como os benefícios advindos com a utilização do mesmo deverão ser criteriosamente avaliados. $O$ autor destaca ainda que em determinadas situações pode-se optar por avaliar o sistema de informações a partir de seus benefícios e aos custos decorrentes de sua adoção. Sob este ponto de vista acredita-se que um sistema de informação sofre influências de seus atributos. [3] Ressalta-se principalmente os atributos relacionados ao tempo, ou seja, a contabilização do registro e da rapidez no processo de recuperação da informação. Outro aspecto a ser considerado é o conteúdo, no que diz respeito ao significado da informação e seu valor agregado percebido pelo usuário para o processo de tomada de decisão. $\mathrm{O}$ aspecto estético, ou a forma em que se apresenta a informação, também, nesta abordagem deve ser observada, bem como o custo. Neste ponto Ahituy, ressalta não ser muito fácil obter o valor exato, destacando a existência de diferentes métodos tais como análises estatísticas e fronteira eficiente. [4] 
Lancaster, relatou em 1995 [5], que referindo-se a Bases de dados em Cd-rom, Jacsó (1992) [6] analisando o gerenciamento da informação, demonstrou que qualquer avaliação de sistemas de informações deve focalizar os aspectos de Hardware, Software e Dataware como aspectos distintos

Diante do exposto, sob nosso ponto de vista, sugerimos considerar que a aceitação de um sistema pelos seus usuários diretos e indiretos, dependa, basicamente, das características, destacadas a seguir: [7]

- valor adicionado', percebido pelos usuários, resultante do uso da informação obtida do sistema, visto sob o aspecto do custo e da importância da informação no processo decisório.

- O uso do sistema que está diretamente relacionado com a Utilidade da informação e facilidade de acesso através do sistema, destacando a existência de dois componentes básicos relacionados ao conteúdo (abrangência e funcionalidade) e facilidade de uso considerando o uso obrigatório por falta de opção, e o uso voluntário função direta da satisfação de usos anteriores.

- custo, que está diretamente relacionado com o uso do sistema, ou seja o nível de investimento realizado pelos usuários durante a utilização do sistema. Esta variável pode ser operacionalizada por diversas visões. Pela visão do usuário final pode ser considerada englobando o custo oportunidade do tempo gasto pelo usuário na aprendizagem do sistema, adicionado ao tempo gasto com o uso propriamente. Pela visão dos analistas de sistemas, programadores e administradores, pode-se considerar o valor despendido com o sistema em termos de hardware, software, recursos humanos, manutenção, e etc ...

- A confiabilidade está diretamente relacionada com a qualidade, que se desdobra nos fatores eficiência, Tempestividade e satisfação do usuário.

É importante notar que os conceitos de utilidade, usabilidade e qualidade, sobre os quais foi definido o presente modelo de avaliação, aplicamse tanto para uma avaliação realizada sob a visão

\footnotetext{
${ }^{1}$ Sob o aspecto de custo trata-se do valor agregado à informação em função dos custos relativos aos processos adicionais que a oneram. Sob o aspecto de qualidade trata-se da importância atribuída à informação no processo de tomada de decisão.
}

de garantia da qualidade de software, quanto sob a visão de qualidade da informação. O foco na satisfação das necessidades do usuário final exige a utilização de variáveis com alto grau de subjetividade. Este esquema conceitual pode ser visualizado através da seguinte figura a seguir:

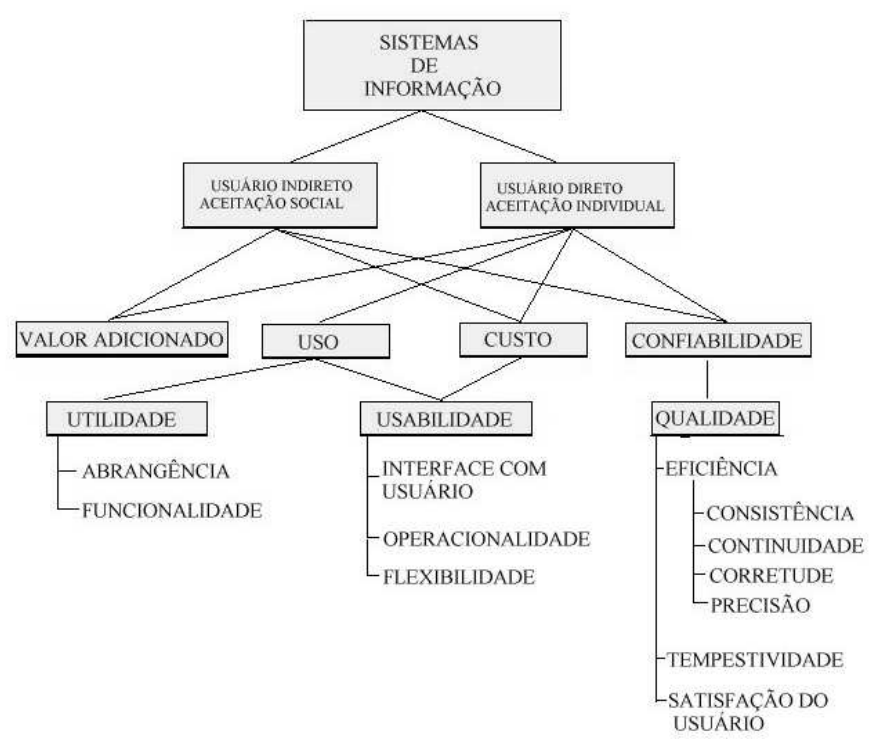

Figura1 - Modelo conceitual para avaliação de sistemas de informação

\section{Resultados : Métricas para Avaliação de Sistemas de Informação.}


O modelo proposto focaliza o comportamento do usuário final centrando a avaliação nas percepções deste quando do uso do sistema, abordando os valores percebidos por estes, sua disposição em usar, o custo e a confiabilidade que 0 sistema oferece. Assim,observa-se que o nível de uso do sistema está associado aos princípios da utilidade, usabilidade e da qualidade, que se desdobram em fatores e subfatores conforme descritos a seguir

\section{Medindo a Abrangência}

Este fator verifica se o software executa todas as rotinas responsáveis pelo seu completo funcionamento. Avalia-se a correspondência entre os procedimentos executados pelo software e as funções descritas como necessárias para cumprir com os objetivos do sistema de informação. Sob a visão da informática, este fator poderá ser quantificado pela razão entre a quantidade de rotinas que foram automatizadas, e que contribuem para 0 atendimento das necessidades do usuário e a quantidade total de rotinas solicitadas pelo usuário.

Sob a visão da Informação, este fator poderá ser quantificado pelo tamanho de sua base de informações, sob o aspecto físico e temporal. O conteúdo é visto por O’Neil em 1988 [8] como um dos muitos fatores que determinam a qualidade de uma base de informações.Em 1990 o grupo de usuários da Southern Califórnia online definiu dez categorias que determinaram a qualidade de bases de informações e de serviços [9]. Mintz em 1990 recomendou abrir espaço aos usuários para comentários que permitam que eles alertem o operados do sistema online sobre os erros encontrados, diretamente, no processo de pesquisa.[10]

\section{Medindo a Funcionalidade}

A qualidade funcional do sistema e o processo usado para desenvolvê-lo pode ser entendida como 0 conjunto de métricas, considerado crítico, para o bom atendimento às necessidades do usuário final. Cada serviço ou modificação requerida em um sistema representa uma necessidade para reparar uma deficiência funcional. A quantidade de correções funcionais, então, pode se transformar em uma métrica interessante que permite visualizar, de uma certa forma, o grau de defeitos do sistema.
As exigências dos usuários finais, cada vez mais conscientes de seu papel junto às instituições, sejam como usuários internos ou externos, contribuem para que os serviços requeridos assumam diversos tamanhos e complexidades. Pontos de controles podem ser utilizados para medir cada requisito ${ }^{2}$ e dar suporte ao impacto que as mudanças terão sobre o sistema todo.

Este fator poderá ser quantificado pela razão entre a quantidade de requisitos atendidos e a quantidade de requisitos solicitados pelo usuário, expurgadas as correções solicitadas.

\section{O Princípio da Usabilidade - Medindo o Fator Usabilidade do Software}

A usabilidade não se limita ao projeto da interface com o usuário [11] [12]. Ela inclui.

- facilidade de uso

- facilidade de reuso:

- eficiência

- poucos erros

- auto aprendizagem

- satisfação e prazer

- percepção favorável do usuário (satisfação do usuário)

A interface deve ser de fácil uso; o acesso às informações deve ser eficiente e deve requerer um mínimo de tempo e esforço dos usuários finais. O sistema de informações deve ser projetado de tal forma que os erros sejam minimizados e próximos de zero. O uso do sistema deve requerer pouco ou nenhum treinamento oferecendo interface intuitiva, permitindo a auto aprendizagem. $O$ aspecto subjetivo da interface é igualmente importante determinando a usabilidade do sistema. A interface deve ser satisfatória para o usuário e o seu uso deve ser prazeroso, isto deve resultar em uma percepção favorável do sistema, pelo seu usuário.

Este fator assegura que o software possua mecanismos que facilitam e viabilizam o uso. Sob a visão da informática poderão ser avaliados os seguintes aspectos:

- se as entradas realizadas pelo usuário são minimizadas por valores pré-definidos;

\footnotetext{
${ }^{2}$ Requisito: requerimentos por parte dos usuários definidos como necessidades a serem atendidas com a operacionalização do sistema
} 
- se existe crítica das entradas antes da realização do processamento;

- se o usuário pode atualizar os dados já fornecidos;

- se o usuário pode selecionar os dispositivos de entradas e/ou saídas;

- se o formato da saída pode ser definido pelo usuário;

- se as saídas são padronizadas e devidamente identificadas por cabeçalhos;

- se existe sistema de ajuda;

- se o sistema de ajuda relaciona as possíveis falhas e apresenta propostas de soluções;

- se a documentação abrange todas as tarefas que o software deve efetuar;

- se os procedimentos descritos no manual são suficientes para executar as tarefas;

- se existe material didático suficiente para treinamento do usuário;

- se o material didático aborda todas as funções do software.

Este subfator pode ser quantificado pela razão entre o número de itens que atendem às especificações de interface humana e a quantidade de itens aplicáveis.

\section{A Tecnologia da Interface com o Usuário}

Nielsen em 1993 traçou a tecnologia disponível para o desenvolvimento de interfaces com o usuário como:

- em lote;

- baseada em linhas;

- tela cheia

- gráfica e

- novas tecnologias

Os sistemas denominados "em lote" são providos com pouca interface para os usuários onde se tem apenas uma chance em cada submissão para requerer trocas ou informações. $O$ uso de arquivos em e-mail e fax é exemplo moderno de interfaces em lote. Nos sistemas baseados em linhas as interfaces permitem que os usuários interajam com o sistema por linhas de comandos, atualmente trocadas pela inclusão de tela cheia com menus hierárquicos.

Nas interfaces gráficas acrescentam-se as representações visuais como abertura de múltiplas janelas para o usuário com interação simultânea com outros sistemas ou fontes de dados.

As tecnologias atuais moveram as interfaces com os usuários em direção a interações com recursos de multimídia e multisensores, tais como animação, vídeo, e áudio, tão bem como dimensões espaciais tais como realidade virtual.

Em 1971, Shackel relatou que cerca de 3\% do orçamento do desenvolvimento de um sistema era destinado para a construção de interfaces permitindo maior grau de usabilidade [13]. Em 1993, Nielsen indicou um percentual entre $6 \%$ e $10 \%$, acrescentando que os usuários finais atribuíam pesos de $30 \%$ para este fator [12]. Estas taxas tendem a crescer à medida que novas tecnologias possibilitam a abertura de novas oportunidades para construção de sistemas de informações mais acessíveis e acessáveis, não só para os técnicos de informação e usuários de computação, mas também para os usuários finais que necessitam das informações, no seu dia a dia para a resolução de seus problemas. Em tais sistemas a qualidade da interface com o usuário é um importante e significativo componente responsável pelo grau de percepção favorável, pelo usuário final, do nível geral de qualidade do sistema.

\section{A Operacionalidade do Sistema}

A operação de um sistema tem mais de uma dimensão. Podemos dividi-la em, pelo menos, duas categorias: o desempenho e a confiabilidade. Apesar destas duas métricas serem relativamente fechadas, e estarem vinculadas ao princípio da qualidade, discutiremos métricas simples para o desempenho e para a confiabilidade. Sob a ótica da operacionalidade, o desempenho do sistema possui as seguintes dimensões.:

- tempestividade;

- eficiência.

A tempestividade de um sistema reflete a rapidez com que uma informação é liberada para os usuários do sistema e no exato momento em que eles dela necessitam. O grau de tempestividade de um sistema deverá ser definido pelos usuários. Isto é extremamente razoável quando consideramos que as necessidades e urgências dos usuários são dinâmicas, ou seja, mudam através do tempo. Na definição de uma métrica de tempestividade há que se estabelecer a diferença entre a disponibilidade da informação para o sistema e a disponibilidade da informação para o usuário. A métrica da tempestividade deve refletir a disponibilidade da informação para os 
usuários. Por exemplo, uma informação pode ser disponibilizada para a atualização de uma base de dados, exatamente no prazo esperado ou planejado para que aconteça a atualização da base. Se ela não for processada dentro do tempo esperado, por dificuldades técnicas ou circunstanciais relacionadas com a base de dados, e a informação não for integrada ao sistema, até que o usuário a utilize, o sistema não poderá ser considerado tempestivo sob a visão dos usuários final. Em geral observa-se a facilidade de navegação, a praticidade da documentação, ou seja todos os aspectos que propiciam menor esforço do usuário para a operação e controle do sistema. Podemos definir uma série de indicadores para medir a tempestividade, dentre outras, citamos:

- o intervalo de tempo entre duas atualizações consecutivas;

- o tempo que o sistema leva para a informação tornar-se disponível para os usuários entre duas atualizações consecutivas;

- tempo de acesso.

A eficiência evidencia que os recursos e os tempos envolvidos para a operação do sistema estejam compatíveis com o nível de desempenho requerido para o produto.

\section{A Flexibilidade do Sistema}

O fator, flexibilidade, define o grau de adaptação do software ao ambiente em que será operacionalizado e a sua utilização em outras plataformas bem como a facilidade de agregação/substituição/desativação de informações e/ou serviços. Podemos avaliar a flexibilidade, sob 0 aspecto da informática, observando se o sistema:

- utiliza solução aberta;

- é multiplataforma;

- é compatível e integrável ao ambiente;

- permite agregar/substituir/desativar com facilidade informações e/ou funções.

Este fator pode ser quantificado pela razão entre o total de itens atendidos e o total de itens observados.

Sob o enfoque da informação, sob o ponto de vista do usuário final, a flexibilidade define o grau de liberdade que o usuário possui para realizar suas consultas à base de informações. Assim, pode-se medir, a flexibilidade, sob este aspecto, avaliando-se, por exemplo:
- quantidade de idiomas em que o sistema permite ser consultado;

- quantidade de campos que podem ser encadeados para restringir o espaço de busca do sistema;

- facilidades de reuso de parte de pesquisas já realizadas, com a retenção de elementos de memória.

Este fator pode ser quantificado pela razão entre a quantidade de itens atendidos e a quantidade de itens aplicáveis.

\section{O Princípio da Qualidade}

O conjunto de requisitos dos usuários finais de sistemas de informação permite-nos obter diferentes componentes nos diversos níveis hierárquicos, na escala de preferências do usuário, e combiná-los para ter um único valor para o sistema, de forma a refletir o grau de confiabilidade dos usuários em relação ao sistema de informações observado.

Os elementos, considerados como requisitos dos usuários, assumem diversos graus de importância de acordo com cada usuário.

Os usuários possuem perfis diferentes e, portanto, não computam o mesmo grau de importância para suas necessidades de informação. Por exemplo, nos ambientes em que existe competição, informações sobre qualidade dos produtos e serviços podem se afigurar com pesos mais altos para os usuários do que informação sobre aproveitamento ou satisfação. Portanto, precisamos identificar as taxas de preferências dos usuários para os vários requisitos dos sistemas de informação.

Qualidade de sistemas de informação em termos de software tem sido a principal preocupação dos desenvolvedores de sistemas e de bases de dados. Uma grande quantidade de modelos com vistas a formalizar o processo de coleta e análise de dados sobre atributos dos sistemas tem sido estudada, pelo menos por duas décadas. Algumas métricas de confiabilidade de software em geral são categorizados em dois tipos principais: aqueles que são projetados para contabilizarem os erros durante os testes e aqueles que consideram como medida de confiabilidade o intervalo de tempo decorrido entre a ocorrência de dois erros. [1].

Os modelos que tratam destas medidas, em geral apresentam-se complexos, e foram analisados e revisados por uma grande 
quantidade de estudiosos, dentre os quais destacamos: Musa [15], Shooman [16], Basili [17], Von Sta [18], Zahedi [14] e Zahedi \& Ashrafi [19].

Falhas no sistema, tais como informação errada, desconexão da linha, queda do sistema ou uma resposta inapropriada, representam respostas não confiáveis. O número de falhas em um determinado intervalo de tempo refere-se ao número de falhas com respeito ao total número de vezes que a unidade foi usada no mesmo intervalo de tempo. O tempo entre duas falhas é a medida de confiabilidade que representa o tempo que leva, para ocorrer duas falhas consecutivas.

A métrica para a confiabilidade do sistema desenvolvida aqui é flexível. Ela requer que as medidas de confiabilidade sejam normalizadas para o intervalo de 0 -1 afim de tornar possível a agregação das várias medidas de confiabilidade.

O sistema de medida de confiabilidade ao nível de entradas, portanto, é a combinação da confiabilidade de cada entrada ponderada pela utilidade relativa que os usuários definiram para cada entrada face as suas necessidades globais de informação. Isto é devido ao fato de que a utilidade relativa pondera as entradas pesos globais que refletem a importância dos componentes relacionados nos níveis prévios. [19]

Este fator poderá ser quantificado considerando-se a utilidade relativa de entradas como o número de vezes que uma determinada entrada produziu saídas confiáveis, dividido pelo total de vezes que a entrada foi utilizada.

\section{Medindo a Eficiência}

O desempenho dos sistemas de informação é particularmente medido pela eficiência do acesso à informação. Pode-se medir a eficiência sob diversas maneiras, conforme segue:

- o tempo que um usuário leva para acessar uma informação;

- o número de comandos, menus, ou ícones que o usuário deve conhecer ou acessar para obter a informação desejada;

- a facilidade de manipular e atualizar o sistema.

- o tempo que um usuário novato leva para aprender a usar o sistema;

- o grau de atendimento às necessidades do usuário, ao nível de conteúdo da informação;

- o volume adequado de informações, segundo as necessidades do usuário final.

A escolha da métrica da eficiência depende da visão sobre o sistema de informação que está relacionada com os objetivos, que nortearam 0 seu desenvolvimento. A eficiência, pode ser desdobrada nos seguintes subfatores:.

\section{Consistência}

A métrica da consistência mostra se 0 desempenho do sistema está de acordo com os interesses de seus usuários. Um sistema que normalmente leva 10 horas para um novato aprender a usá-lo é preferível a outro que leva 36 horas. Uma métrica para a consistência dos sistemas de informação é o desvio padrão de outra métrica. Por exemplo, considere o tempo que um usuário leva para acessar uma informação como a métrica para eficiência. $O$ sistema deverá ser observado em um período de tempo que esteja em uso por vários usuários que tentam acessar diferentes informações. Existirá um conjunto de dados para o tempo de acesso. Computando a média deste conjunto de dados obter-se-á o valor da métrica para a eficiência do sistema. O desvio padrão mostra a consistência da eficiência do sistema. [19]

$$
\alpha=\sum_{\mathrm{i}=1}^{\mathrm{n}} \frac{\underline{\mathbf{a}}_{\mathrm{i}}}{\mathbf{n}}
$$

Onde : $\boldsymbol{\alpha}=$ Média do tempo de acesso

$\mathbf{a}_{\mathbf{i}}=$ Tempo de acesso observado

$\mathbf{n}=$ Quantidade de dados sobre os tempos de acessos considerados.

Assim considerando que $\alpha$ é a métrica para a eficiência do sistema, temos que a consistência do sistema será obtida por:

$$
\delta=\sum_{i=1}^{n}\left(\frac{\left(\underline{a}_{i}-\tilde{a}\right)^{2}}{n-1}\right)
$$

Onde: $\boldsymbol{\delta}=$ desvio padrão da série de dados que representam os tempos de acesso.

Assim $\delta$ pode ser considerada como a medida de consistência do sistema, em tempo de acesso.

\section{Continuidade}

A continuidade do serviço do sistema é a ininterrupção na liberação de seus serviços. Uma métrica para esta dimensão de performance é a percentagem de tempo em que o sistema esteve 
indisponível para seus usuários. A métrica da continuidade pode ser obtida por:

$$
\alpha=\sum_{i=1}^{u} \frac{t_{i}}{T}
$$

Onde: $\mathbf{u}$ =quantidade de dados que representam os intervalos de tempos em que o sistema ficou indisponível no período t.

$\mathbf{T}=$ período de tempo em que o sistema ficou em observação

$\mathbf{t}_{\mathbf{i}}=$ tamanho da indisponibilidade do sistema em ti/h que o sistema ficou indisponível.

O objetivo de zero defeito força esta métrica e seu desvio padrão em direção para zero

\section{Corretude}

A corretude pode ser definida como sendo informações completas e precisas sem defeitos. Considerando esta definição temos que a métrica para corretude, sob a visão da informática, inclui:

- quantidade de defeitos descobertos depois que o sistema entrou em operação;

- quantidade de defeitos descobertos e relatados pelos usuários finais;

- intervalo de tempo em que o especialista de informática levou para ajustar um defeito relatado;

- quantidade de defeitos causados pelo ajuste de outros defeitos.

Esta métrica deve ser definida para um intervalo de tempo ou deve ser normalizada. É útil no gerenciamento do projeto de desenvolvimento de sistemas de informação, especificamente no gerenciamento de falhas.

Sob a visão da informação, podemos definir a métrica para a corretude como sendo:

- mensagens condizentes com as ações desencadeadas pelo sistema;

- volume de informações de acordo com o grau de especificação definido pelo usuário final;

- precisão no conteúdo das informações, informações sem erros de conteúdo.

A corretude pode ser categorizada dentro das seguintes medidas:

- média do número de erros de dados de entrada dentro do sistema (sobre o número total de dados entrados) em determinado intervalo de tempo;
- média do número de informações que são incorretamente processadas (sobre todas as informações processadas);

- média do número de vezes que uma informação acessada e estava desatualizada (sobre o total de informações atualizadas no sistema) em determinado intervalo de tempo.

- média de solicitações de usuários para as quais o sistema foi incapaz de responder (sobre todas as solicitações do sistema) em um determinado intervalo de tempo;

- média do número de vezes em que a informação foi transmitida erradamente sobre linhas de comunicação (sobre todas as informações transmitidas) em um dado intervalo de tempo.

Enquanto a corretude é computada como sendo uma média sobre um intervalo de tempo, a consistência dessa métrica de corretude é computada como sendo um desvio padrão dessa mesma métrica e naquele mesmo intervalo de tempo. A definição da consistência é similar à definição adotada para a média e desvio padrão dos tempos de acessos, quando tratamos da apuração da consistência.

O problema principal com métricas de desempenho é que elas são computadas para vários componentes do sistema. Nenhuma proporciona uma métrica global para o sistema, como um todo, como ele é visto pelos seus usuários finais.

\section{Precisão}

Este subfator possibilita assegurar se 0 sistema opera corretamente por um período de tempo, no Hardware para o qual foi projetado. Sob a visão da informática, podemos avaliar a precisão de um sistema de informação, observando-se :

- se o software possui mecanismos de autoproteção contra situações adversas e tratamento de entradas incorretas mantendo-se em operação normal;

- se existe crítica dos dados de entrada;

- se o processamento dos dados é executado somente após a validação dos dados;

- se o software possui meios de tratar problemas de falha de leitura/gravação, fim de arquivo inesperado, underflow, overflow, divisão por zero, e outros. 
O que melhor expressa esta característica é a taxa de erro, e é importante mantê-la no ponto mínimo. Quanto mais exata a informação, maior será sua contribuição para a tomada de decisão. É comum se dizer que quanto maior a exatidão da informação, maior será o custo em obter, verificar e manter os dados nos quais ela se baseia.

Para observar este fator devemos distinguir a informação sob dois aspectos: relativo aos procedimentos realizados pelo software e relativo ao valor das informações derivadas pelo processo. A precisão relativa aos procedimentos do software assegura que o software proporcione um nível de exatidão nos cálculos, pesquisas e resultados de modo a satisfazer às necessidades do usuário. Quantifica-se este fator através da média aritmética das seguintes medidas:

- a razão entre a quantidade de processos, que não consideram os requisitos de precisão para as entradas, o processamento e as saídas e a quantidade total de processos;

- a razão entre a quantidade de algoritmos que não tiveram a exatidão comprovada por análise matemática e o total de algoritmos existentes;

- razão entre a quantidade de rotinas utilizadas sem comprovação de atendimento aos requisitos de precisão e a quantidade total de rotinas.

Sob a visão da informação, é importante notar que o grau requerido de precisão varia de acordo com o uso destinado à informação. A informação baseada em projeções será certamente menos precisa do que aquela baseada em dados históricos. Da mesma forma estimativa de longo prazo será menos precisa que a de curto prazo.

Para os métodos de inferência convencional, a ausência total de informação é melhor do que informação cujo grau de imprecisão a torna dispensável, uma vez que neste caso as regras SE...E...ENTÃO... são baseadas em respostas rígidas do tipo $\mathrm{Sim} / \mathrm{Não}$. Distinção se faz no caso de fuzzy inferência, onde além de decisões do tipo Sim/Não, também são permitidos valores intermediários. Neste segundo caso, considerando contextos complexos, a característica precisão é menos relevante que no caso de inferência convencional. Assim posto, torna-se difícil quantificar os efeitos das informações junto ao processo de tomada de decisões.

\section{Medindo a Tempestividade}

A informação deve estar disponível quando necessária, de outra forma se tornará de pouca ou nenhuma utilidade. Assim, precisamos também considerar o tempo necessário à busca e ao processamento do dado ou da informação.

A observação deste fator assegura que a solução apresentada atende a todas as necessidades do usuário com bom desempenho.

Pode-se avaliar a Tempestividade, observando-se por exemplo:

- se o tempo de resposta atende às expectativas do usuário;

- Se a solução atende a todas as necessidades do usuário;

- Se existe plano de contingência;

- Se o sistema funciona sem interrupção por problemas de software.

Este fator pode ser quantificado pela razão entre o total de itens atendidos e o total de itens aplicáveis.

\section{Medindo a Satisfação do Usuário}

Qualquer critério só será eficiente para medir a qualidade de um sistema de informação, se o usuário julgá-lo importante. $O$ indicador de qualidade representa a eficácia com que o processo que está sendo avaliado atende às necessidades dos usuários. Assim, o caminho mais curto para se elaborar critérios para medir a qualidade é conhecer a opinião do usuário com relação aos seus produtos e serviços e transformar essa avaliação em indicadores.

\section{Grau de Satisfação do Usuário.}

Qual deve ser a abrangência de uma pesquisa sobre satisfação dos usuários? satisfação do usuário determina como os usuários se sentem em relação a produtos e serviços.

A pesquisa, não deve intimidar os respondentes, devendo se possível ser anônima, e proporcionar um clima propício para que os usuários usem o maior grau de honestidade para responderem. $\mathrm{O}$ levantamento deve assegurar respostas que deverão ser utilizadas somente para dar suporte à aplicação identificando-se oportunidades para melhorias.

Existem várias formas de cálculo para se obter o nível de conhecimento do grau de satisfação dos usuários finais podemos citar como 
exemplo os modelos inspirados no processo de planejamento estratégico com Identificação de Fatores Críticos de sucesso, desenvolvidos pelos prof. Alloway e Quillard, do Massachusetts Instituto of Technology, Cambridge, Mass, USA. [20].

Estas formas podem ser bem simples, desde a pesquisa pura e simples sobre qual pontuação o usuário atribuiria á determinado sistema de informação considerando uma escala de 0 a 10, até pesquisas que permitem ao usuário final emitir sua opinião sobre o grau de desempenho do sistema, segundo determinados fatores pré-estabelecidos e o grau de importância desses fatores.

Este método é uma adaptação do processo de identificação dos fatores críticos de sucessos, para elaboração do planejamento estratégico de sistemas de informações gerenciais.

Descreveremos, a seguir, duas sugestões para a avaliação e cálculo do grau de satisfação dos usuários finais.

\section{Comparação entre o Nível de Utilização do Sistema e o Grau de Satisfação do Usuário.}

A comparação será observada através de gráficos e da montagem de um modelo matemático onde serão considerados os graus de influência de cada fator no nível de satisfação do usuário, e o seu peso quando comparado com o grau de utilização da base de dados.

\section{Pesquisa Interna}

As empresas devem solicitar ajuda às organizações especializadas para realizar tais pesquisas. Uma palavra inadequada utilizada em uma pergunta tornará os resultados inválidos. A amostra de usuários deverá ser determinada observando-se critérios estatísticos, rigorosos, devendo possuir características o suficiente para representar, o universo de usuários e possibilitar uma análise adequada. A empresa poderá proceder à pesquisa eletronicamente ou através de questionários. Cabe ressaltar que as pesquisas conduzidas eletronicamente garantirão resultados mais rapidamente e permitirão esclarecer quaisquer dúvidas mais rapidamente.

\section{Agregação das Métricas.}

Os valores apurados deverão ficar entre 0 e 1, aproximando-se de 1 quanto mais adequado ao uso o sistema for. O processo de agregação das métricas, por ser objetivo, permite a quantificação dos fatores e a agregação da pontuação . Assim os valores encontrados a média aritmética dos fatores Abrangência e Funcionalidade deverão compor o fator Utilidade.

As médias aritméticas dos subfatores Usabilidade, Flexibilidade e Tempestividade, deverão compor o fator Acessibilidade, e a média aritmética dos subfatores satisfação do usuário , Confiabilidade e Precisão, corresponderá ao valor do fator Qualidade. A média dos fatores Utilidade, Acessibilidade e Qualidade, corresponderá ao grau de perfeição alcançado com o desenvolvimento do aplicativo. Os valores dos indicadores variarão entre 0 e 1 , aproximando-se de 1 à medida que o produto ou serviço atinja a perfeição.

\section{Um Modelo para Avaliação de Utilização de Sistemas de Informação.}

Baseados nas três dimensões discutidas nas seções anteriores, resumindo, definimos a Utilidade do sistema como a satisfação e os benefícios percebidos pelo usuário derivados do uso do sistema.

A Usabilidade do sistema, por outro lado, refere-se a medida que a interface do sistema se adequa aos seus usuários no tocante às suas necessidades e desejos, e a Qualidade está relacionada à confiabilidade das informações e ao resultado obtido com o uso dela. Acreditamos que os fatores externos associados, especificamente à qualidade do sistema, enquanto produto, bem como os fatores associados às necessidades de informação do usuário conduzem um usuário a utilizar ou não um sistema de informações. Se esses estímulos são positivos, o usuário fica propenso a utilizar o sistema, e contribui para um possível estado de plena utilização. Caso contrário, se os estímulos são recebidos pelo usuário de forma negativa, o usuário desistirá de utilizar o sistema, e continuará sua busca pela informação em fontes alternativas, contribuindo para um estado de subutilização do sistema de informação. Esta proposição pode ser visualizada pela figura 2, a seguir: 


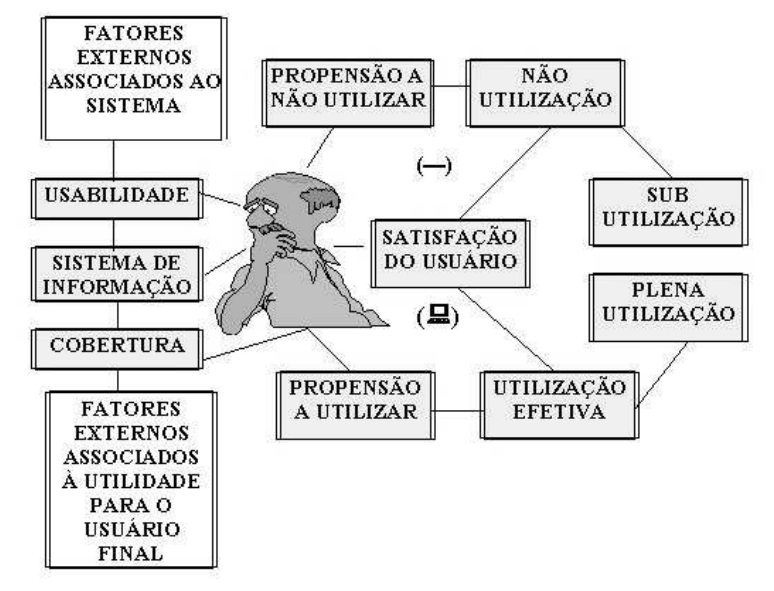

Figura 2 - Esquema do comportamento do usuário

\section{Princípio da Utilidade}

Associado aos fatores que determinam 0 nível de abrangência do sistema em relação ao atendimento às necessidades funcionais do usuário final.

\section{Princípio da Usabilidade}

Associado aos fatores que determinam a facilidade de operacionalização do sistema permitindo 0 acesso à informação $e$

\section{Princípio da Qualidade}

Associado aos fatores que determinam a o nível de satisfação do usuário final em relação aos produtos e serviços da área de sistemas.

Para Grudin, a utilização de sistemas de informação tem dois componentes: utilidade e usabilidade [21]. Já Nielsen em 1993 distingue utilidade e usabilidade da seguinte forma: utilidade está relacionada à funcionalidade do sistema ou seja se o sistema realiza o que se espera que ele realize, e usabilidade relaciona-se com a questão de quão bem os usuários podem usar aquela funcionalidade. Nesta definição o conceito de usuários não está limitado para os usuários finais, eles incluem os que têm que instalar, manter e aperfeiçoar o sistema [12].

Ao depararmo-nos com a necessidade de informações para resolução de problemas, buscamos nos sistemas automatizados as informações que precisamos. Este primeiro passo baseia-se no princípio da utilidade, pois partimos em busca de informações que nos serão úteis no processo de tomada de decisão. Em nível mais detalhado, consideramos os fatores associados à abrangência do sistema como responsáveis pelo atendimento das necessidades do usuário. Ou seja, consideramos a abrangência da fonte de pesquisa, e dos procedimentos implementados no sistema, como primeiro indicativo de que poderemos obter as informações desejadas. Destacamos como variáveis básicas para cobertura do princípio da Utilidade a abrangência e a funcionalidade.

Passamos, então, à segunda questão: Como obter as informações que precisamos? passamos à identificação das variáveis que estariam relacionadas com o princípio "Facilidade de acesso à informação". Consideramos então, os critérios associados à Usabilidade, onde destacamos os fatores inerentes ao software: flexibilidade, facilidade de uso e de qualidade da interface com o usuário.

O terceiro princípio, o da Qualidade, permite-nos responder à questão: quão satisfeitos com o produto e com a informação obtida, estão os usuários?

Neste aspecto é importante considerar o grau de conhecimento sobre informática ou mesmo sobre o nível de envolvimento do usuário com a equipe de desenvolvimento durante a fase de construção do sistema. É importante frisar que o domínio da informática, relacionado a comandos de alto nível, permitindo a geração de relatórios, influencia sobremaneira, no nível de satisfação do usuário final.

A busca por um método simples e realista para avaliação do impacto e o valor percebido de um sistema junto ao usuário final, é de suma importância para a definição das variáveis ligadas 
à utilização, que se referem ao sistema como facilidade de uso, acessibilidade, e ligadas ao usuário como utilidade que está relacionada ao conteúdo, e a qualidade da informação obtida. Estes aspectos estão intimamente ligados à propensão do usuário a utilizar ou não determinado sistema, e garantem a avaliação do sucesso ou fracasso de um sistema de informações.

As aplicações liberadas para o usuário devem ser projetadas de forma a atingir alto grau de usabilidade, portanto devem oferecer, ao usuário final, documentação e treinamentos adequados. Aplicações subutilizadas, em geral, são mal documentadas, ou são executadas por usuários mal treinados. Estas deficiências se refletirão em demandas para o centro de ajuda e/ou equipe de suporte e manutenção. Grande número de chamadas indica uma necessidade de mudança na aplicação e/ou de reformulação da documentação ou necessidade de treinamento do usuário. A análise das solicitações permitir-nos-ão identificar se haverá ou não necessidade de reengenharia da interface para o usuário.

Altos níveis de chamadas implicam em uma nova implementação; o declínio em chamadas indica que as causas das chamadas são resultantes de falhas de treinamento e experiência com o sistema .

O princípio da Acessibilidade está associado aos fatores: Usabilidade, flexibilidade e tempestividade. A Acessibilidade pode ser quantificada pela média aritmética do total da pontuação atribuída aos fatores Usabilidade, flexibilidade e tempestividade.

\section{Conclusões}

A proposta constitui-se em um modelo, para avaliação da qualidade de sistema de informação. Os critérios são objetivos, e a avaliação focaliza o usuário final como elemento essencial para o delineamento das metas de qualidade dos produtos e serviços.

Apresenta, ainda, um plano de testes para avaliação da qualidade do software, focalizando os aspectos que influenciam o comportamento do usuário final. Para a utilização dos resultados da observação do plano de avaliação do sistema em relação aos usuários caracterizados como analistas de sistemas, programadores e administradores de bases de dados, o controle da qualidade pressupõe a existência de um ambiente de Engenharia de Software e exige um comportamento disciplinado durante 0 desenvolvimento do software. Os indicadores de qualidade permitem além de obter-se o nível de utilização do sistema em questão, para o momento posterior à avaliação, proporciona uma visão do grau de eficiência do processo de desenvolvimento de software além de identificar oportunidades de melhorias e o grau de aceitação do sistema em relação aos seus usuários diretos e indiretos.

\section{Referências Bibliogáficas}

1. DAVIS, F.D. Perceive use fulness, perceived ease of use, and user acceptance of information technology. MIS Quarterly, p.319339, Sept.1989.

2. NEWELL, a \&IMON, H. A. Human problema solving. Englewood Cliffs, Prentice-Hall, 1972.

3. AHITUY, N. Principles of information system for management. 3nd.ed. Dubuque, EUA: WCB, 1990. $653 \mathrm{p}$.

4. FREITAS, Henrique M. R. et al. Avaliação de sistemas de informações. Revista de Administração, São Paulo, v. 29, n.4, p 36-55, out/dez 1994.

5. LANCASTER, F. Wilfrid et al. Evaluation of interactive knowledge - based systems: overview and design for empirical testing. Journal of the American Society for Information Science.[S.I.],v.47,n.1p.57-69, 1996..

6. JACSÓ, P. CD-ROM software, dataware and hardware: evaluation, selection, and installation. Englewood, CO: Libraries Unlimited, 1992.

7. Mendes, Raquel Dias - Um modelo simplificado para avaliação do nível de utilização de sistemas de informação. UNB, 1999

8. O'NEILL, E., VIZINE-GOETZ, D. Quality controls in online databases. Annual review of information science and technology.[S.I.],n.23,p.125-156,1988.

9. $\mathrm{BASCH}$, Reva. Database realiability: The black box. New York, Medford (ed), 1990.

10. MINTZ, Anne P. Quality Control and the zen of database production. Online.[S.I.],v.14,n.6,p.15-23,Nov.[199-].

11. LAND, F. Adapting to changing user requirements, Information \& Management, 5(1), pp.91-107, 1982. 
12. NIELSEN, Jacob. Usability engineering. New York: Academic Press, 1993.

13. SHACKEL, B. Human factors in the P.L.A Meat Handling Automation Scheme: a case study and some conclusions. International Journal of Production Research,[S.I.],v.9,n.1,p.95-121,1971.

14. Zahedi, F., Quality information systems: an International Thomson Publishing Company. Wisconsin: University of Wisconsin, 1995.

15. MUSA, J. D. A theory of software reliability and its application, IEEE transactions on software engineering.[S.I.:s.n.]:v.1, p. 312327,1971.

16. SHOOMAN, M.L Probabilistic models for software reliability prediction. In:FREIBERGER,W. Statistical computer performance evaluation. New York: Ed. Academic, 1972.p.485-502.

17. BASILI , V. R. Tutorial on models and metrics for software management and engineering. Washington, DC., IEEE Computing Society, 1980.

18. VON STAA, Arnd. Garantia da qualidade de software. Rio de Janeiro: PUC, 1989.

19. ZAHEDI, F., ASHRAFI, N. Software reliability allocation based on structure, utility, price, and Cost. IEEE Transactions on software engineering,[S.I.],v.17,n.4,p.345-356,Apr.1991.

20. ALLOWAY, Robert, M., QUILLARD, Judith. An integrated definition of DP Success and its managerial implications. [S.I.:s.n.], 1980.

21. GRUDIN, J. Utility and usability: research issues in development contexts. Interacting with Computers.[S.I.],v.4,n.2, p. 209-217, Aug.1992.

\section{Contato}

Profa. Dra. Raquel Dias

e-mail: raquel dias@directnet.com.br raquel dias@yahoo.com

Consultora independente em Tecnologia da Informação, Economista, Doutora em Ciência da Informação pela UNB, mestre em informática pelo ITA, Pós Graduada em: Inteligência Competitiva pela UFRJ, Gerência Empresarial e Engenharia Econômica pelo ICAT/DF e em Informática e Análise de Sistemas pela PUC/DF. 